\title{
Coordination-Induced Band Gaps in MOFs
}

\author{
Craig A. Peeples ${ }^{a}$, Ahmet Çetinkaya ${ }^{b, g}$, Franz-Josef Schmitt ${ }^{c}$, Patrik Tholen ${ }^{d}$, Yunus Zorlu ${ }^{\mathrm{e}}$, Kai Bin \\ $\mathrm{Yu}^{\mathrm{f}}$, Ozgur Yazaydin ${ }^{f}$, Jens Beckmann ${ }^{\mathrm{b} *}$, Gabriel Hanna ${ }^{\mathrm{a} *}$, Gündoğ Yücesan ${ }^{\mathrm{d} *}$
}

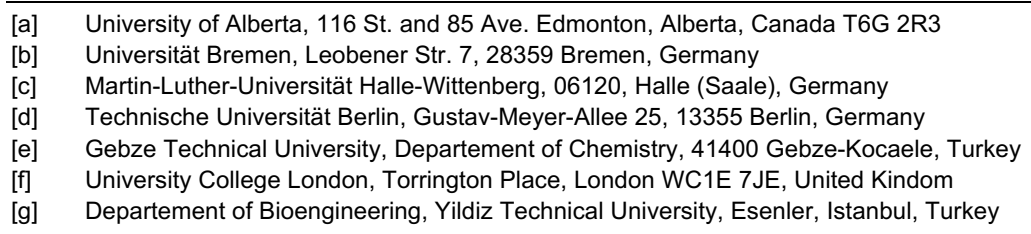

Supporting information for this article is given via a link at the end of the document.

\begin{abstract}
:
Herein, we report on the synthesis of a microporous, threedimensional phosphonate metal-organic framework (MOF) of the composition $\mathrm{Cu}_{3}\left(\mathrm{H}_{5}-\mathrm{MTPPA}\right)_{2} \cdot 2$ NMP $\left(\mathrm{H}_{8}-\mathrm{MTPPA}=\right.$ methane tetra- $p$-phenylphosphonic acid and NMP $=\mathrm{N}$-methyl-2pyrrolidone). This MOF, termed TUB1, has a unique onedimensional inorganic building unit composed of square planar and distorted trigonal bipyramidal copper atoms. It possesses a (calculated) BET surface area of $766.2 \mathrm{~m}^{2} / \mathrm{g}$ after removal of the solvents from the voids. The Tauc plot for TUB1 yields indirect and direct band gaps of $2.4 \mathrm{eV}$ and $2.7 \mathrm{eV}$, respectively. DFT calculations reveal the existence of two spin-dependent gaps of $2.60 \mathrm{eV}$ and $0.48 \mathrm{eV}$ for the alpha and beta spins, respectively, with the lowest unoccupied crystal orbital for both gaps predominantly residing on the square planar copper atoms. The projected density of states suggests that the presence of the square planar copper atoms reduces the overall band gap of TUB1, as the beta-gap for the trigonal bipyramidal copper atoms is $3.72 \mathrm{eV}$.
\end{abstract}

\section{Introduction}

Metal-organic framework (MOF) research has gained significant momentum over the last twenty years. ${ }^{1-3}$ To date, thousands of MOF crystal structures have been deposited into the crystal structure databases. Their use in diverse applications range from small molecule storage, ${ }^{4-7}$ over catalysis, ${ }^{8-10}$ magnetism, ${ }^{11-15}$ conductivity, ${ }^{16,}{ }^{17}$ solar cells, ${ }^{18}$ drug delivery, ${ }^{19,} 20$ to food chemistry $^{21}$, etc. MOFs provide a synthetically flexible platform ${ }^{22-}$ 24 and synthesized MOFs may undergo structural optimizations via defect engineering ${ }^{25}$ and post-synthetic modifications. ${ }^{26,27}$ Consequently, MOFs possess greater structural flexibility and a wider range of applications compared to traditional high surface area materials such as zeolites, carbon nanotubes ${ }^{28,29}$, and graphene. ${ }^{30-32}$ One of the emerging applications in MOF chemistry is the development of electrically conductive electrode materials for supercapacitor applications. ${ }^{16,33}$ The large surface areas and structural flexibility/diversity of MOFs provide unique advantages for optimizing the conductivity and charge-holding capacities of next-generation supercapacitors. For example, several MOFs are known to possess very high surface areas exceeding $7000 \mathrm{~m}^{2} / \mathrm{g}$ compared to graphene or activated carbon electrodes. ${ }^{34,35}$ However, since current MOF chemistry has evolved around well- separated molecular inorganic building units (IBUs) and wellseparated organic struts to increase pore surface areas, ${ }^{24,36}$ this approach inhibits electron-hopping mechanisms for electrical conduction and reduces the magnetic interactions between the metal ions. ${ }^{37}$ This is why conventional MOFs constructed using arylcarboxylic acid linkers are generally known to be insulators. ${ }^{16,38}$ Guest incorporation in carboxylate MOFs has been shown to be effective in making insulating MOFs conductive..$^{39-41}$ MOFs containing well-aligned two-dimensional sheets constructed using azolate, ${ }^{42}$ ortho-diimine,${ }^{43-47}$ quinoids, ${ }^{48}$ orthodihydroxy, ${ }^{49}$ and sulfur-donating ${ }^{50}$ linkers have been shown to be highly electrically conductive (see Ref. 16 for comprehensive lists of such MOFs). Such linkers are chelators with conserved metalbinding modes, which promote the formation of conductive twodimensional sheets, e.g., ortho-diimine and ortho-dihydroxy MOFs form via the chelation of single square planar metal atoms. As a result, such MOFs generally possess a single type of IBU, which limits their structural diversity and ability to optimize their surface areas and conductivities. ${ }^{16,33,51,52}$

In addition to the two-dimensional conductive MOFs, we have recently shown that arylphosphonic acid linkers with molecular, one-, and two-dimensional IBUs can produce electrically conductive three-dimensional MOFs via electron-hopping and extended conjugation mechanisms. In fact, we recently reported the highest single-crystal conductivity for a three-dimensional MOF using arylphosphonic acid linkers. ${ }^{37,53,54}$ Phosphonate MOFs possess extremely rich metal-binding modes compared to any other MOF family in the literature, which give rise to diverse IBUs in one and two dimensions. ${ }^{55-57}$ Phosphonate MOFs with one-dimensional IBUs generally form one-dimensional void channels surrounded by well-stacked aromatic units, ${ }^{58}$ while those with two-dimensional IBUs form pillared-layered networks. ${ }^{55}$ It is believed that the well-layered aromatic units in such phosphonate MOFs help promote electron hopping and that the continuous arrangement of M-O-P polyhedra in the IBUs extend the conjugation. ${ }^{37,53}$ Moreover, due to their exceptionally high thermal and chemical stabilities, phosphonate MOFs could be suitable for applications such as electrodes in supercapacitors for electric vehicles and the storage/supply of energy produced by solar panels and wind turbines. ${ }^{12,59-61}$ In this work, we report the crystal structure of a newly synthesized phosphonate MOF, termed TUB1, which possesses a unique one-dimensional IBU composed of square planar and distorted trigonal bipyramidal copper(II) ions, and a tris-deprotonated methane tetra- $p$ phenylphosphonic acid linker. TUB1 was found to have a calculated BET surface area of $766.2 \mathrm{~m}^{2} / \mathrm{g}$, and an indirect band gap of $2.4 \mathrm{eV}$. 


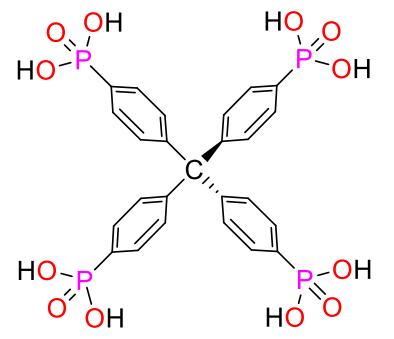

Scheme 1. Solvothermal reaction for synthesis of TUB1
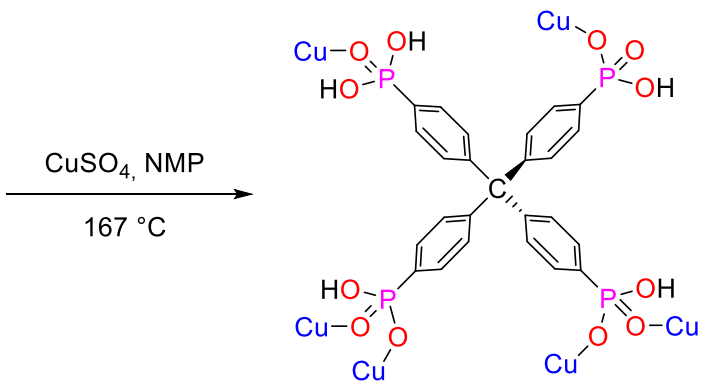

\section{Results and Discussion}

The solvothermal synthesis of TUB1 involved the reaction between $\mathrm{CuSO}_{4}$ and the $\mathrm{H}_{8}$-MTPPA linker (synthesized according to our previously reported method ${ }^{12}$ ) in a polar aprotic N-methyl2-pyrrolidone (NMP) solvent at $167{ }^{\circ} \mathrm{C}$ using Teflon-lined $43 \mathrm{ml}$ Parr acid digestion vessels (Scheme 1). To ensure that the $\mathrm{H}_{8-}$ MTPPA linker did not fully deprotonate (in order to restrain its metal-binding modes), we adapted our previously employed $\mathrm{pH}$ controlled reaction conditions. ${ }^{12,54,62}$ Using this methodology, we obtained light blue single crystalline plates of the exact composition $\mathrm{Cu}_{3}\left(\mathrm{H}_{5}-\mathrm{MTPPA}\right)_{2} \cdot 2$ NMP (see Figure 1$)^{63}$ with a trisdeprotonated $\left[\mathrm{H}_{5}-\mathrm{MTPPA}\right]^{3-}$ linker in ca. $2 \%$ average yield and 98\% $\mathrm{H}_{8}$-MTPPA crystals. Different synthetic conditions with different solvents did not improve the yield as the crystallization of the $\mathrm{H}_{8}$-MTPPA linker was always preferred. TUB1 is composed of one-dimensional IBUs, which are bridged together by $\left[\mathrm{H}_{5}-\right.$ MTPPA $]^{3-}$ linkers. Each IBU is composed of corner-sharing eightmembered Cu1-O-P-O-Cu1-O-P-O and Cu2-O-P-O-Cu2-O-P-O rings. Since three of the four phosphonic acid units in the linker are mono-deprotonated, the linker has asymmetric metal-binding modes, viz., the mono-deprotonated units could give rise to ionic interactions while the fully protonated unit provides coordinate covalent binding. Therefore, TUB1 exhibits two different metalbinding options, in contrast to our previously reported $\mathrm{CO}_{2}\left(\mathrm{H}_{4-}\right.$ MTPPA) and $\mathrm{Zn}_{2}\left(\mathrm{H}_{4}-\mathrm{MTPPA}\right)$ MOFs. ${ }^{12,64}$ In particular, the three mono-deprotonated phosphonic acid units are coordinated to square planar and distorted trigonal bipyramidal copper centers (labelled Cu1 and Cu2, respectively) via ionic bonding, while the fully protonated unit exclusively generates coordinate covalent bonding between the square planar copper center and the $\mathrm{P}=\mathrm{O}$ bond of the phosphonic acid. (The density functional theory (DFT)-based charge analysis in Table S6 shows that there is high electron delocalization on the deprotonated oxygens, suggesting the possibility of ionic interactions.) To the best of our knowledge, the presence of both trigonal bipyramidal and square planar copper centers in a one-dimensional IBU has not been previously reported for phosphonate MOFs. ${ }^{58}$ The crystal structure of TUB1 indicates the presence of two different types of square- and parallelogram-shaped void channels (see Figure 1). From Grand Canonical Monte Carlo simulations, the BET surface area and Helium-accessible pore volume of TUB1 were estimated to be $766.2 \mathrm{~m}^{2} / \mathrm{g}$ and $0.296 \mathrm{~cm}^{3} / \mathrm{g}$, respectively (see SI for details). Next, the indirect and direct band gaps of TUB1 were estimated from Tauc plots of the UV-Vis spectrum (see Figure 2) to be $2.4 \mathrm{eV}$ and $2.7 \mathrm{eV}$, respectively. The Tauc plots of $\mathrm{H}_{8}-\mathrm{MTPPA}$ reveal direct and indirect band gaps of ca. $4.2 \mathrm{eV}$, which are considerably wider than those of TUB1 and contain two jumps as opposed to one in the TUB1 plots. This is likely due to the fact that $\left[\mathrm{H}_{5}-\mathrm{MTPPA}\right]^{3-}$ has an insulating $\mathrm{sp}^{3}$ methane core. The lower band gap of TUB1 arises from the formation of one-dimensional IBUs composed of eight-membered Cu1-O-P-O-Cu1-O-P-O and Cu2-O-P-O-Cu2$\mathrm{O}-\mathrm{P}-\mathrm{O}$ rings in the MOF. Such a dramatic change in the Tauc plot after the formation of TUB1 suggests the emergence of an electron hopping mechanism, as the $\mathrm{sp}^{3}$ methane core in $\left[\mathrm{H}_{5}-\right.$ MTPPA $]^{3-}$ blocks the extension of conjugation in three dimensions.

A thermal gravimetric analysis (TGA) of TUB1 was performed to examine its thermal behavior. As seen in Figure S6, at ca. $100^{\circ} \mathrm{C}$, the remaining water molecules evaporate from the structure. NMP starts to leave the pores of TUB1 at ca. $200^{\circ} \mathrm{C}$ (in accordance with its boiling point at $202^{\circ} \mathrm{C}$ ).
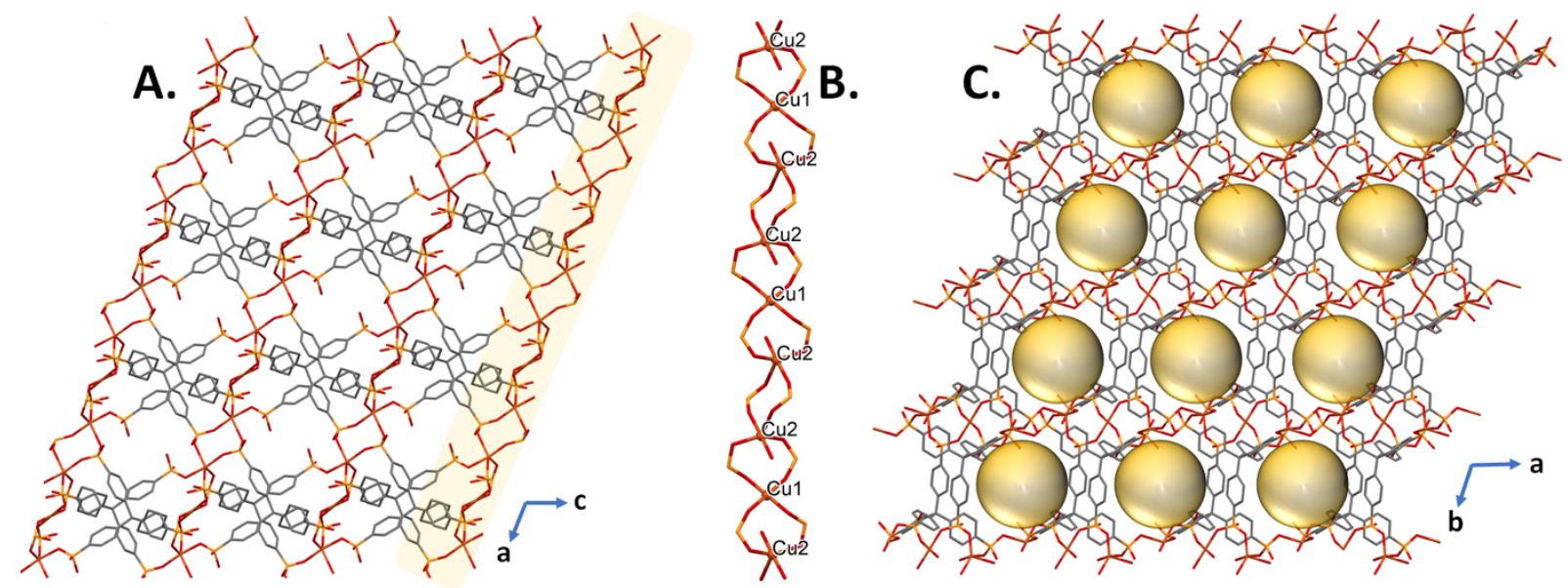

Figure 1. a) View of the rod shaped inorganic building unit (highlighted in yellow) in the ac plane of TUB1 b) Structure of the one-dimensional IBU of TUB1 c) View of parallelogram void channels in the ab plane of TBU1. NMP molecules were omitted. 

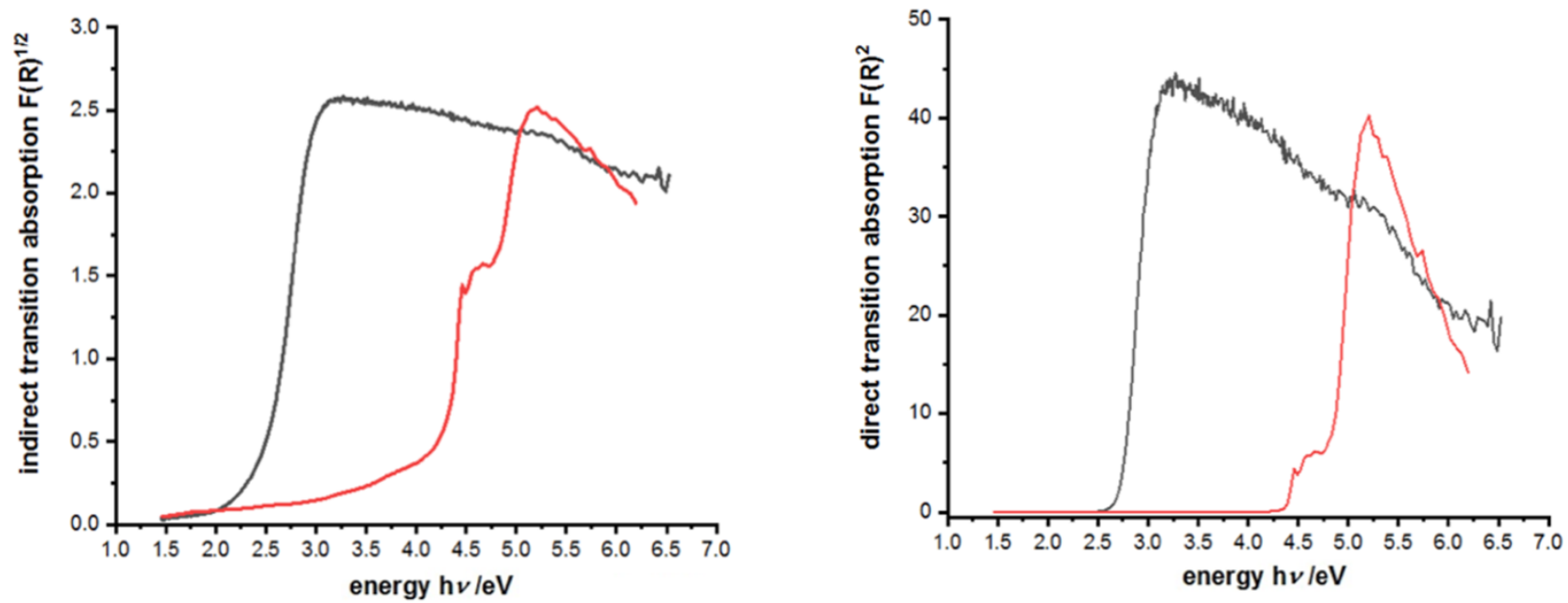

Figure 2. Tauc plots of UV-Vis spectra used to obtain the indirect (left) and direct (right) band gaps of TUB1 (black) and the H8-MTTPA linker (Red).

The $\left[\mathrm{H}_{5}-\mathrm{MTPPA}\right]^{3-}$ organic components start decomposing at ca. $420^{\circ} \mathrm{C}$ and the decomposition pattern remains stable until $850^{\circ} \mathrm{C}$, suggesting the formation of thermally stable phosphide clusters. ${ }^{68}$ This pattern is similar to those of previously reported copper phosphonates. ${ }^{62,69}$

To gain deeper insight into the electronic structure of TUB1, we performed DFT calculations of the highest occupied crystal orbital (HOCO)-lowest unoccupied crystal orbital (LUCO) gap, projected density of states (pDOS), HOCO and LUCO, average partial charges, and band structure. The details of our DFT calculations using Slater-type orbitals (STOs) and the HSE $06^{70}$ functional may be found in the SI. The optimized structures of a $2 \times 2 \times 2$ supercell and a single unit cell of TUB1 are shown in Figure 3, with the spin polarizations of each copper atom indicated on the unit cell. The structural differences between the optimized structure and experimental crystal structure are small (see Table S3). As illustrated in Figure 4, the copper atoms in the unit cell have different coordination environments, with the $\alpha$-copper atoms (i.e., copper atoms with an excess of spin-up electrons) in the trigonal bipyramidal geometry and the $\beta$-copper atom in the square planar geometry. Next, the pDOS was calculated using the rangeseparated hybrid functional HSE06, ${ }^{70}$ which is known to yield accurate solid-state HOCO-LUCO gaps. ${ }^{71,72}$ An analysis of the pDOS (shown in Figure 5) reveals a potential electrical conduction mechanism involving two spin-dependent HOCO-LUCO gaps, viz., a spin-up ( $\alpha$ ) gap of $2.60 \mathrm{eV}$, which is in good agreement with the experimental estimates of $2.4 \mathrm{eV}$ (indirect) and $2.7 \mathrm{eV}$ (direct), and a spin-down ( $\beta$ ) gap of $0.48 \mathrm{eV}$. As seen in the pDOS, the $\beta$ HOCO-LUCO gap, which lies beneath the Fermi energy, is generated by orbitals primarily on the carbon atoms and some on the oxygen and copper atoms. From the $\beta$-HOCO and $\beta$-LUCO iso-surfaces in the top panel of Figure 6 , we see that the $\beta$-HOCO is primarily composed of $\pi$-orbitals on the $\mathrm{sp}^{2}$ carbon atoms in half of the phenyl rings down the b-axis. The $\beta$-LUCO is composed of the same phenyl carbon $\pi$-orbitals, as well as $\pi$-orbitals on the oxygen atoms and d-orbitals on the square planar copper atoms (see Figures S3 and S4). The higher energy $\beta$-unoccupied crystal orbitals are primarily composed of orbitals on the trigonal bipyramidal copper atoms and yield a gap of $3.72 \mathrm{eV}$ (see Figure S3). 
A.

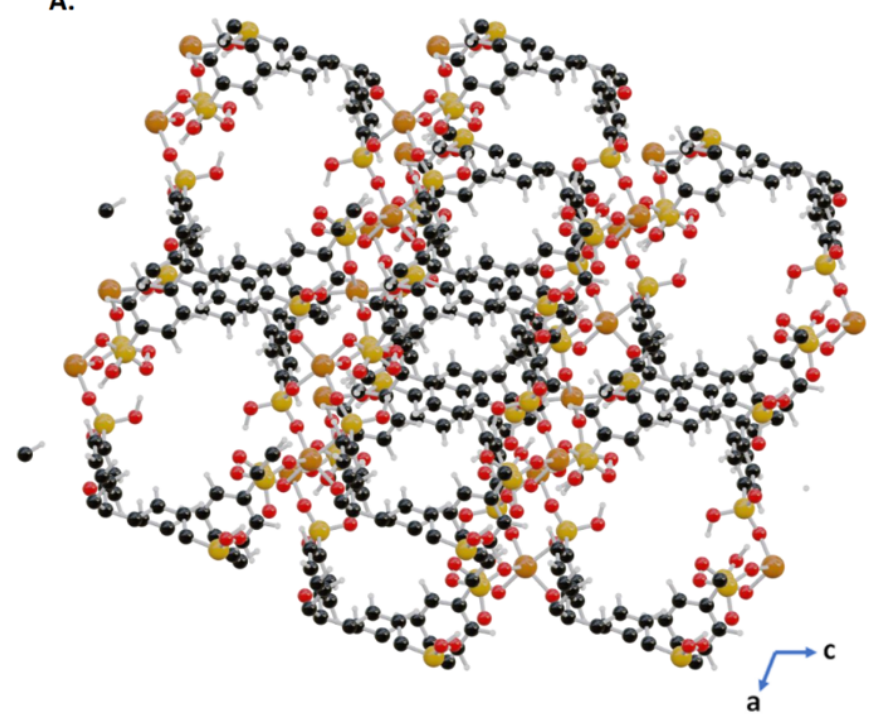

B.

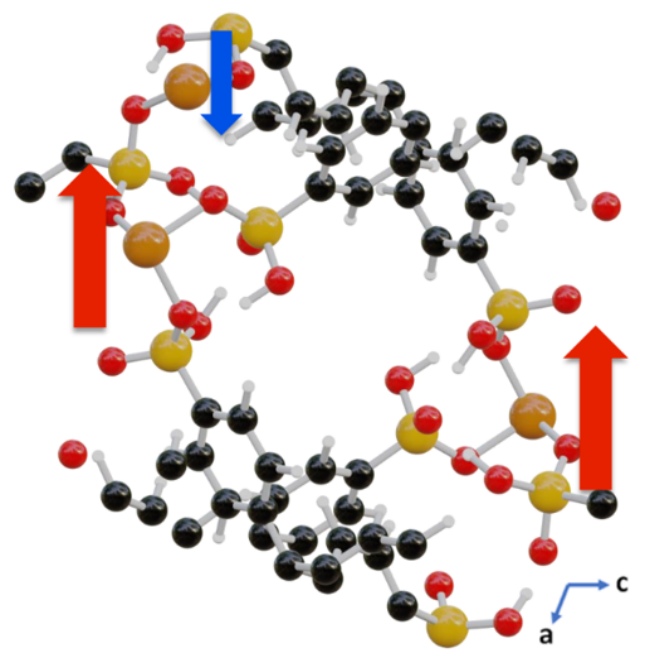

Figure 3. Optimized structure of TUB1. a) A view down the b-axis of the $2 \times 2 \times 2$ supercell. b) A view down the b-axis of the unit cell, with the red (spin-up) and blue (spin-down) arrows indicating the minimum-energy spin configuration of the unpaired electrons on the copper atoms (Cu - light brown; $\mathrm{P}-\mathrm{yellow}$; $\mathrm{O}-$ red; $\mathrm{C}-$ black; $\mathrm{H}-$ white).

A.

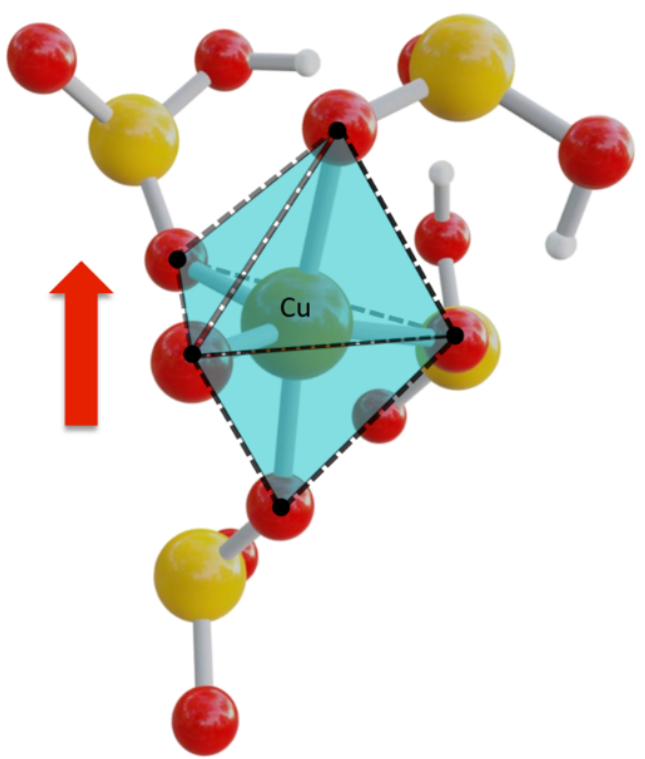

B.

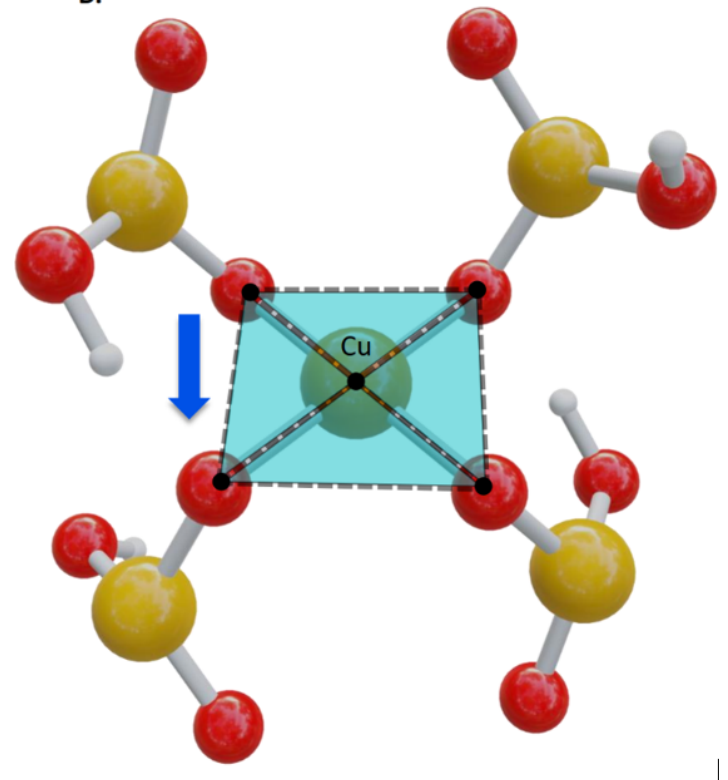

Figure 4. A portion of the TUB1 unit cell, highlighting the two types of copper coordination environments. a) Trigonal bi-pyramidal copper atom coordinated to five oxygen atoms. b) Square planar copper atom coordinated to four oxygen atoms ( $\mathrm{Cu}$ - light brown central atom; $\mathrm{P}-\mathrm{yellow}$; $\mathrm{O}-$ red; $\mathrm{H}-$ white).

Comparing this value to the $\beta$ - and $\alpha$-HOCO-LUCO gaps of 0.48 $\mathrm{eV}$ and $2.60 \mathrm{eV}$, respectively, generated by square planar copper atoms, it is evident that the coordination environment of the copper atoms plays an essential role in narrowing the HOCOLUCO gap. From the $\alpha-H O C O$ and $\alpha$-LUCO iso-surfaces in the bottom panel of Figure 6 , we see that the a-HOCO is composed of carbon and oxygen $\pi$-orbitals along the $b$-axis, while the $a$ LUCO is primarily composed of d-orbitals on the square planar copper atom (see Figures S3 and S4) and m-orbitals on the oxygen and carbon atoms. The participation of the carbon, oxygen, and square planar copper atoms in both spin-dependent HOCO-LUCO gaps suggests that they are integral in facilitating electrical conduction in TUB1. The band structure of TUB1 is shown in Figure S5. As can be seen, there are $120 \mathrm{meV}$ dispersions in the $\beta$-LUCO band along the $X-\Gamma, \Gamma-Y, L-\Gamma, \Gamma-Z, N-$ $\Gamma, \Gamma-\mathrm{M}$, and $\mathrm{R}-\Gamma$ paths in k-space. This points to higher electron mobility in several directions compared to typical semiconducting MOFs, which have linear bands or bands with dispersions of less than 50 meV. ${ }^{16,73}$ 


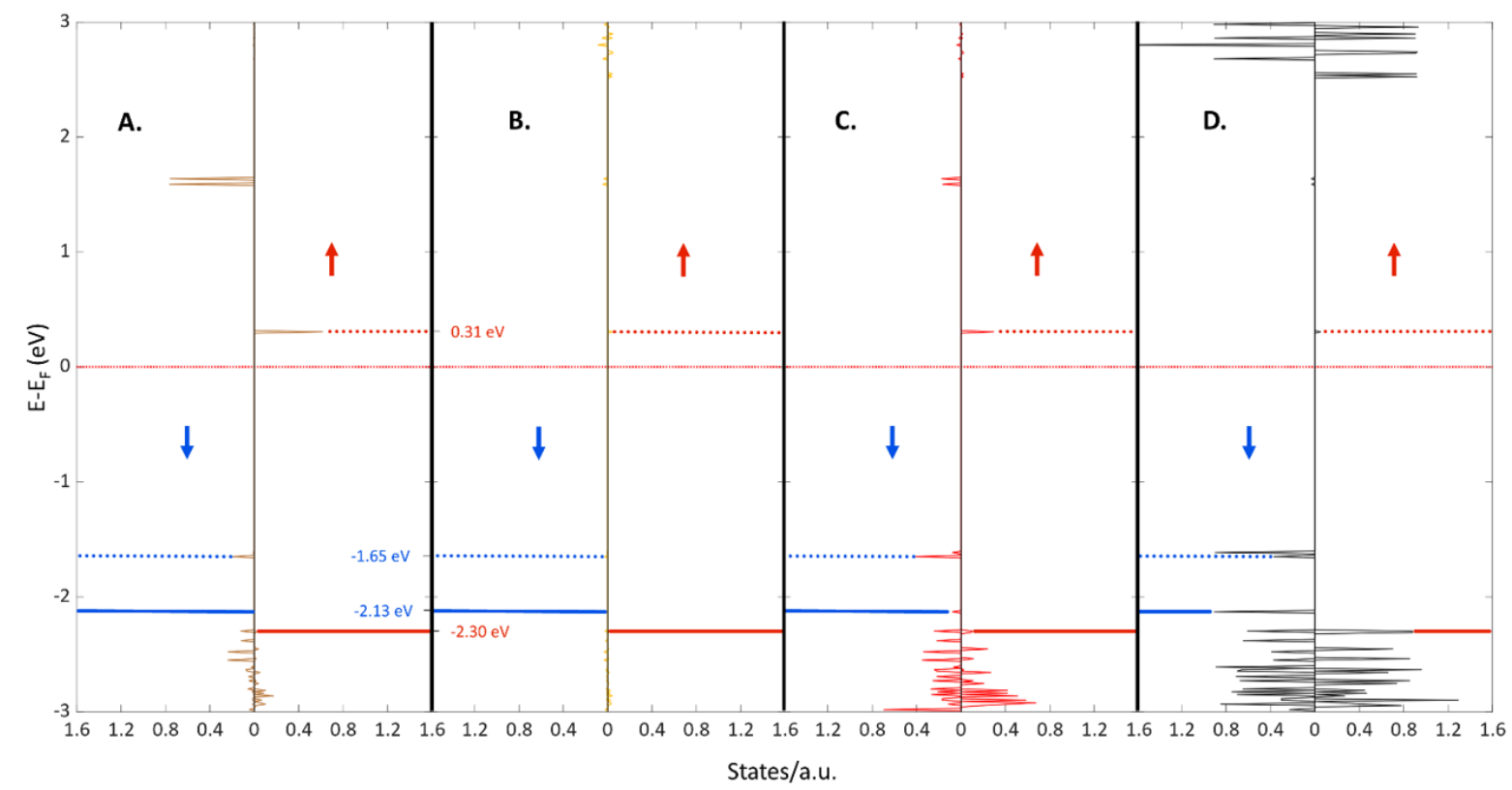

Figure 5. Spin-up (red arrow) and spin-down (blue arrow) projected density of states for TUB1: (A) Copper, (B) Phosphorous, (C) Oxygen, (D) Carbon. The solid red and blue lines indicate the $\alpha$ - and $\beta$-HOCO energy levels, respectively. The dotted red and blue lines indicate the $\alpha$ - and $\beta$-LUCO energy levels, respectively. The blue numbers in panel A indicate the energies of the $\beta$-HOCO and $\beta$-LUCO, while the red numbers in panel B indicate the energies of the $\alpha-\mathrm{HOCO}$ and $\alpha-$ LUCO.
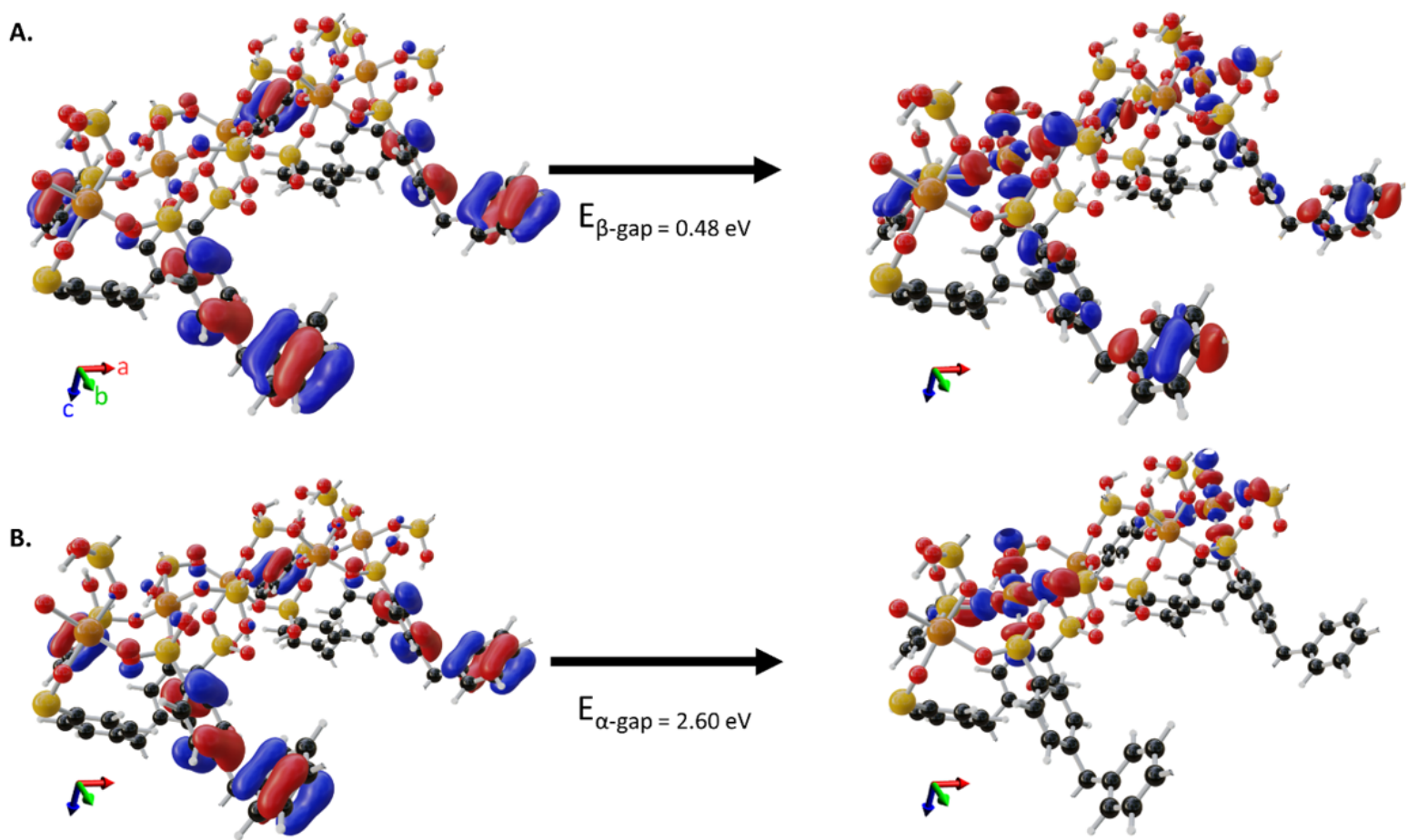

Figure 6. HOCO and LUCO iso-density surfaces, corresponding to a density of 1 electron per $0.03 \AA^{3}$. A) $\beta$-spin HOCO-LUCO gap. B) $\alpha$-spin HOCO-LUCO gap. For clarity, only a portion of the unit cell is shown ( $\mathrm{Cu}$ - light brown; $\mathrm{P}$ - yellow; $\mathrm{O}$ - red; $\mathrm{C}$ - black; $\mathrm{H}$ - white).

\section{Conclusion}

In summary, we have reported on the synthesis and electronic properties of a low-gap three-dimensional MOF TUB1, which possesses a unique one-dimensional IBU composed of cornersharing eight-membered Cu1-O-P-O-Cu1-O-P-O and Cu2-O-P$\mathrm{O}-\mathrm{Cu} 2-\mathrm{O}-\mathrm{P}-\mathrm{O}$ rings containing square planar and distorted trigonal bipyramidal copper centers. TUB1 was found to have a
BET surface area of $766.2 \mathrm{~m}^{2} / \mathrm{g}$ and Helium-accessible pore volume of $0.296 \mathrm{~cm}^{3} / \mathrm{g}$ (as predicted by Monte Carlo simulations), and an indirect band gap of $2.4 \mathrm{eV}$. Our DFT calculations confirm the experimental structure, yield spin-dependent HOCO-LUCO gaps of $2.60 \mathrm{eV}(\alpha)$ and $0.48 \mathrm{eV}(\beta)$, suggest a role of the square planar copper centers in reducing the HOCO-LUCO gap, and point to above-normal non-directional electron mobility. The uniqueness of TUB1's IBU was made possible by the structural diversity afforded by phosphonate metal-binding groups. Such metal phosphonate IBUs may be used to construct 
semiconductive MOFs with polyaromatic cores for use in nextgeneration supercapacitors.

\section{Acknowledgements}

G.Y. would like to thank the DFG for funding his work with grant number DFG YU 267/2-1 and J.B. would like to thank the DFG for funding his work with grant number BE 3716/9-1. G.H. acknowledges funding from the Natural Sciences and Engineering Research Council of Canada (NSERC). The DFT calculations were enabled by support provided by WestGrid (www.westgrid.ca) and Compute Canada (www.computecanada.ca).

\section{Abbreviations}

MOF, metal-organic framework; $\mathrm{H}_{8}-\mathrm{MTPPA}$, methane tetra- $p$ phenylphosphonic acid; IBU, inorganic building unit; NMP, Nmethyl-2-pyrrolidone; TUB1, Technische Universität Berlin 1; DFT, density functional theory; $\mathrm{HOCO}$, highest occupied crystal orbital; LUCO, lowest unoccupied crystal orbital; pDOS, projected density of states; STO, Slater-type orbital; HSE06, HeydScuseria-Ernzerhof 06; TGA, thermo gravimetric analysis.

\section{Conflict of Interest}

The authors declare no conflict of interest.

Keywords: Metal Organic Framework (MOF), Ligand Design, Solvothermal Synthesis, Density of States, Surface Area

1. M. J. Kalmutzki, N. Hanikel, O. M. Yaghi, Sci Adv 2018, 4,10 .

2. J. L. C Rowsell, O. M. Yaghi, Micropor. Mesopor. Mater. 2004, 73, 3 .

3. J. D. Evans, B. Garai, H. Reinsch, W. Li, S. Dissegna, V. Bon, I. Senkovska, R. A. Fischer, S. Kaskel, C. Janiak, N Stock, D. Volkmer, Coord. Chem. Rev. 2019, 380, 378-418.

4. P. Z. Moghadam, A. Li, X. W. Liu, R. Bueno-Perez, S. D. Wang, S. B. Wiggin, P. A. Wood, D. Fairen-Jimenez, Chem. Sci. 2020, 11, 8373.

5. Li, A.; Bueno-Perez, R.; Wiggin, S.; Fairen-Jimenez, D., Enabling efficient exploration of metal-organic frameworks in the Cambridge Structural Database. CrystEngComm. 2020, 22, 7152.

6. G. Férey, C. Serre, T. Devic, G. Maurin, H. Jobic, P. L. Llewellyn, G. De Weireld, A. Vimont, M. Daturi, J.-S. Chang, Chem. Soc. Rev. 2011, 40, 550-562.

7. A. Schneemann, V. Bon, I. Schwedler, I. Senkovska, S. Kaskel, R. A. Fischer, Chem. Soc. Rev. 2014, 43, 6062-96.

8. D. Yang, B. C. Gates, ACS Catal. 2019, 9, 1779.

9. A. Corma, H. García, F. X. Llabrés i Xamena, Chem. Rev. 2010, 110, 4606.

10. L. Ma, J. M. Falkowski, C. Abney, W. Lin, Nat. Chem. 2010, 2, 838.

11. D. Riou, F Belier, C. Serre, M. Noguès, D. Vichard, G. Férey, G., Int. J. Inorg. Mater. 2000, 2, 29.

12. Y. Zorlu, Y. D. Erbahar, A. Çetinkaya, A. Bulut, T. S. Erkal, A. O. Yazaydin, J. Beckmann, G. Yücesan, Chem. Commun. 2019, 55, 3053-3056.
13. W. Ouellette, A. V. Prosvirin, K. Whitenack, K. Dunbar, J. Zubieta, Angew. Chem., Int. Ed. 2009, 48, 2140.

14. Z. Wang, K. Hu, S. Gao, H. Kobayashi, Adv. Mater. 2010, 22, 1526 .

15. A. E. Thorarinsdottir, T. D. Harris, Chem. Rev. 2020, 120, 8716-8789.

16. L. S. Xie, G. Skorupskii, M. Dincă, Chem. Rev. 2020, 120, 8536-8580.

17. L. Sun, M. G. Campbell, M. Dincă, Angew. Chem., Int. Ed. Engl 2016, 55, 3566-79.

18. C-C. Chueh, C.-I Chen, Y.-A Su, H. Konnerth, Y.-J Gu, C.W Kung, K. C. W. Wu, J. Mater. Chem. A 2019, 7, 1707917095.

19. S. Rojas, A. Arenas-Vivo, P. Horcajada, Coord. Chem. Rev. 2019, 388, 202-226.

20. P. Horcajada, T. Chalati, C. Serre, B. Gillet, C. Sebrie, T. Baati, J. F. Eubank, D. Heurtaux, P. Clayette, C. Kreuz, J.S. Chang, Y. K. Hwang, V. Marsaud, P.-N. Bories, L. Cynober, S.; Gil, G. Férey, P. Couvreur, R. Gref, Nat. Mater. 2010, 9, 172-178.

21. N. Manousi, G. A. Zachariadis, E. A. Deliyanni, V. F. Samanidou, Molecules 2018, 23 (11).

22. Furukawa, H.; Cordova, K. E.; O'Keeffe, M.; Yaghi, O. M., Science 2013, 341 (6149), 1230444.

23. H. C. Zhou, J. R. Long, O. M. Yaghi, Chem. Rev. 2012, 112, 673-4.

24. O. M. Yaghi, M. J. Kalmutzki, C. S. Diercks, Weinheim: Wiley-VCH, 2019.

25. M. Taddei, Coord. Chem. Rev. 2017, 343, 1-24.

26. M. Kim, J. F. Cahill, Y. Su, K. A. Prather, S. M. Cohen, Chem. Sci. 2012, 3, 126.

27. S. M. Cohen, J. Am. Chem. Soc. 2017, 139, 2855.

28. L. T. Scott, E. A. Jackson, Q. Y. Zhang, B. D. Steinberg, M. Bancu, B. Li, J. Am. Chem. Soc. 2012, 134, 107.

29. B. Xiao, P. S. Wheatley, R. E. Morris, R. Xu, Z. Gao, J. Chen, W. Yan, From Zeolites to Porous MOF MaterialsThe 40th Anniversary of International Zeolite Conference. 2007; p 902.

30. L. Jiang, Z. Fan, Nanoscale 2014, 6, 1922.

31. M. J. Allen, V. C. Tung, R. B. Kaner, Chem. Rev. 2010, 110, 132.

32. A. J. Clancy, H. Au, N. Rubio, G. O. Coulter, M. S. P. Shaffer, Dalton Trans. 2020, 49 (30), 10308-10318.

33. D. Sheberla, J. C. Bachman, J. S. Elias, C. J. Sun, Y. Shao-Horn, M. Dincă, Nat. Mater. 2017, 16, 220.

34. O. K. Farha, I. Eryazici, N. C. Jeong, B. Hauser, C. E. Wilmer, A. A. Sarjeant, R. Q. Snurr, S. T. Nguyen, A. Yazaydın, J. T. Hupp, J. Am. Chem. Soc. 2012, 134, 1501621.

35. I. M. Hönicke, I. Senkovska, V. Bon, I. A. Baburin, N. Bönisch, S. Raschke, J. D. Evans, S. Kaskel, Angew. Chem., Int. Ed. Engl. 2018, 57, 13780-13783.

36. O. M. Yaghi, M. O'Keeffe, N. W. Ockwig, H. K. Chae, M. Eddaoudi, J. Kim, Nature 2003, 423, 705.

37. K. Siemensmeyer, C. A. Peeples, P. Tholen, F. J. Schmitt, B. Çoşut, G. Hanna, G. Yücesan, Adv. Mater. 2020, 32 (24), 202000474.

38. M. Taddei, G. M. Schukraft, M. E. A. Warwick, D. Tiana, M. J. McPherson, D. R. Jones, C. Petit, J. Mater. Chem. A 2019, 7, 23781-23786.

39. C. Schneider, D. Ukaj, R. Koerver, A. A. Talin, G. Kieslich, S. P. Pujari, H. Zuilhof, J. Janek, M. D. Allendorf, R. A. Fischer, Chem. Sci. 2018, 9, 7405.

40. K. Thurmer, C. Schneider, V. Stavila, R. W. FriddleF. Leonard, R. A: Fischer, M. D. Allendorf, A. A. Talin, ACS Appl. Mater. Interfaces 2018, 10, 39400.

41. A. A. Talin, A. Centrone, A. C. Ford, M. E. Foster, V. Stavila, P. Haney, R. A. Kinney, V. Szalai, F. El Gabaly, H. P. Yoon, F. Léonard, M. D: Allendorf, Science 2014, 343 (6166), 66. 
42. L. Xie, L. Sun, R. Wan, S. S. Park, J. A. DeGayner, C. H. Hendon, M. Dincă, J. Am. Chem. Soc. 2018, 140, 7411.

43. D. Sheberla, J. C. Bachman, J. S. Elias, C. J. Sun, Y. Shao-Horn, M. Dinca, Nat. Mater. 2017, 16, 220.

44. T. Chen, J. H. Dou, L. Yang, C. Sun, N. J. Libretto, G Skorupskii, J. T. Miller, M. Dincă, J. Am. Chem. Soc. 2020, 142, 12367.

45. M. G. Campbell, D. Sheberla, S. F. Liu, T. Swager, M. Dincă, Angew. Chem., Int. Ed. 2015, 54, 4349.

46. G. Skorupskii, B. A. Trump, T. W. Kasel, C. M. Brown, C. H. Hendon, M. Dincă, Nat. Chem. 2020, 12, 131.

47. D. Sheberla, L. Sun, M. A: Blood-Forsythe, S. Er, C. R. Wade, C. K.Brozek, A. Aspuru-Guzik, M. Dincă, J. Am. Chem. Soc. 2014, 136, 8859.

48. J. A. DeGayner, I. R. Jeon, L. Sun, M. Dincă, T. D: Harris, J. Am. Chem. Soc. 2017, 139, 4175.

49. R. W. Day, D. K. Bediako, M. Rezaee, L. R. Parent, G. Skorupskii, M. Q. Arguilla, C. H. Hendon, I. Stassen, N. C Gianneschi, P. Kim, M. Dincă, ACS Central Science 2019, 5, 1959-1964.

50. Y. Kobayashi, B. Jacobs, M. D: Allendorf, J. R. Long, Chem. Mater. 2010, 22, 4120.

51. M. G. Campbell, D. Sheberla, S. F. Liu, T. Swager, M. Dincă, Angew. Chem., Int. Ed. 2015, 54, 4349.

52. R. W. Day, D. K. Bediako, M. Rezaee, L. R. Parent, G. Skorupskii, M. Q. Arguilla, C. H. Hendon, I. Stassen, N. C. Gianneschi, P. Kim, M. Dincă, ACS Cent. Sci. 2019, 5, 1959-1964.

53. C. A: Peeples, D. Kober, F. J. Schmitt, P. Tholen, K. Siemensmeyer, Q. Halldorson, B. Çoşut, A. Gurlo, A. O. Yazaydin, G. Hanna, G. Yücesan, Adv. Funct. Mater. 2021 31, 2007294

54. Y. Zorlu, P. Tholen, M. M. Ayhan, B. C., Bayraktar, G. Hanna, A. O. Yazaydin, G. Yücesan, 2021, https://doi.org/10.26434/chemrxiv.13653050.v1

55. G. Yücesan, Y. Zorlu, M. Stricker, J. Beckmann, Coord. Chem. Rev. 2018, 369, 105-122.

56. R. A. Coxall, S. G. Harris, D. K. Henderson, S. Parsons, P. A. Tasker, R. E. P. Winpenny, J. Chem. Soc., Dalton Trans. 2000, 2349-2356.

57. D. Sahoo, R. Suriyanarayanan, V. Chandrasekhar, Dalton Trans. 2014, 43, 10898-10909.

58. P. Tholen, Y. Zorlu, J. Beckmann, G. Yücesan, Eur. J. Inorg. Chem. 2020, 1542-1554.

59. Y. -P. Zhu, J. Yin, E. Abou-Hamad, X. Liu, W. Chen, T. Yao, O. F. Mohammed, H. N. Alshareef, Adv. Mater. 2020, 32 (16), 1906368.

60. C. Y: Gao, J. Ai, H. R. Tian, D. Wu, Z. M. Sun, Chem. Commun. 2017, 53, 1293-1296.

61. T. Zheng, Z. Yang, D. Gui, Z. Liu, X. Wang, X. Dai, S Liu, L. Zhang, Y. Gao, L. Chen, D. Sheng, Y. Wang, J. Diwu, J. Wang, R. Zhou, Z. Chai, T. E. Albrecht-Schmitt, S. Wang, Nat. Commun. 2017, 8, 15369.

62. M. M. Ayhan, C. Bayraktar, K. B. Yu, G. Hanna, A. O Yazaydin, Y. Zorlu, G. Yücesan, Chem. Eur.J. 2020, 26 14813-14816.

63. Depositon number 1973476 contains the supplementary crystallographic data for this paper. These data are provided free of charge by the joint Cambridge Crystallographic Data Centre and Fachinformationszentrum Karlsruhe Access Strucure service.

64. A. Schütrumpf, A. Bulut, N. Hermer, Y. Zorlu, E. Kirpi, N. Stock, A. O. Yazaydın, G. Yücesan, J. Beckmann, ChemistrySelect 2017, 2, 3035-3038.

65. J. Park, A. C. Hinckley, Z. Huang, G. Chen, A. A. Yakovenko, X. Zou, Z. Bao. J. Am. Chem. Soc. 2020, 142, 20531-20535.

66. G. Chen, L. B. Gee, W. Xu, Y. Zhu, J. S. Lezama-Pacheco, Z. Huang, Z. Li, J .T. Babicz, S. Choudhury, T. H. Chang, E. Reed, E. I, Solomon, Z. Bao, J. Am. Chem. Soc. 2020, 142 21243-21248

67. A. Yadav, D. Panda, S. Zhang, W. Zhou, S. Saha, ACS Appl.
Mater. Interfaces 2020, 12, 40613-40619.

68. R. Zhang, P. A. Russo, M. Feist, P. Amsalem, N. Koch, N Pinna, ACS Appl. Mater. Interfaces 2017, 9, 14013-14022.

69. Y. Y. Enakieva, A. A. Sinelshchikova, M. S. Grigoriev, V. V. Chernyshev, K. A. Kovalenko, I. A. Stenina, A. B. Yaroslavtsev, Y. G. Gorbunova, A. Y. Tsivadze, Chem. Eur. J. 2019, $25,10552-10556$.

70. J. Heyd, G. E. Scuseria, J.Chem. Phys. 2006, 124, 219906.

71. A. J. Garza, G. E. Scuseria, J. Phys. Chem. Lett. 2016, 7, 4165-4170.

72. J. P. Perdew,. et al. Proc. Natl. Acad. Sci. U. S. A. 2017, 114 , 2801-2806.

73. A. Pathak, J. W. Shen, M. Usman, L. F. Wei, S. Mendiratta Y. S. Chang, B. Sainbileg, C. M. Ngue, R. S. Chen, M. Hayashi, T. T. Luo, F. R. Chen, K. H. Chen, T. W. Tseng, L. C. Chen, K. L. Lu, Nat. Commun. 2019, 10, 1-7. 
